\title{
Für eine bessere Epidemiologie der Jodmangelstruma
}

Hypothesen unterscheiden sich von Gebilden der willkürlichen Phantasie dadurch, daß sie auf gesichertem Wissen aufbauen, mit kleinen oder großen Sprüngen neue Wahrheiten anvisieren und dabei in absehbarer Zukunft nachprüfbar sein sollen. Unsere Hypothese ist:

- Die Diskussion um endemische Struma und Jodmangel in der Bundesrepublik Deutschland erfaßt bisher nur die Spitzen eines gewaltigen Eisberges.

Folgende Überlegungen und Fragen müssen hierbei diskutiert werden:

1. Gründlichere, das heißt neuere und mit höherer regionaler Auflösung durchgeführte epidemiologische Untersuchungen über die alimentäre Jodversorgung sind für die Bundesrepublik Deutschland höchst wünschenswert. So paßt Ostholstein zum Beispiel hinsichtlich des alimentären Jodmangels zu den benachbarten Regionen Mecklenburg, Dänemark, Niedersachsen und östliche Niederlande.

2. Bisherige epidemiologische Daten über die Strumahäufigkeit bei Schulkindern oder Rekruten ergeben ein unrichtiges Bild. Für Kinder gibt es keine Normalwerte der Schilddrüsengröße (sonographische Volumetrie); palpatorisch wird vielleicht zu oft eine Schilddrüsenvergrößerung angenommen. Das höhere Alter muß in diese Untersuchungen eingeschlossen werden, da sonst die zunehmende Prävalenz übersehen wird.

3. Die Grenzen der Methodik der Strumaerkennung selbst sind besser zu definieren. Die Richtigkeit der palpatorischen und der sonographischen Größenbestimmung bleibt besser zu belegen, und die differentialdiagnostischen Irrtumsmöglichkeiten bei strumaverdächtigen Röntgenbefunden lassen Sensitivität und Spezifität dieses Verfahrens beim Einsatz als epidemiologische Methode noch kaum erkennen.

Dtsch. med. Wschr. 108 (1983), 1007

(c) 1983 Georg Thieme Verlag Stuttgart - New York
4. Selbstverständlich müssen sich auch Epidemiologen bezüglich der Stichprobenwahl nach dem "Möglichen " richten. Beispielsweise mögen in den schweren Endemiegebieten Tansanias (Jodmangelstruma und Kretinismus) Schulkinder und junge Mütter als erste, wichtigste Zielgruppe zunächst ausreichen. Für die Bundesrepublik Deutschland ist es heute schon schwierig bis unmöglich, Schulkinder oder Rekruten ohne Selektion zu untersuchen. Der Datenschutz erschwert eine so einfache Frage wie die Ermittlung des Geburtsortes aus Röntgenreihenuntersuchungen. Durch solche zusätzlichen Hemmnisse wird die epidemiologische Erforschung der Prävention behindert.

5. Die Stichprobenauswahl muß mit großer Vorsicht zur Vermeidung von selektionsbedingten Trugschlüssen und Verallgemeinerungen erfolgen. Zum Beispiel muß die Strumahäufigkeit bei der arbeitenden Bevölkerung nicht mit derjenigen der Gesamtbevölkerung übereinstimmen.

6. Führt eine unzureichende Jodprophylaxe der Struma zu einer Verschiebung der Strumamanifestation in das höhere Lebensalter, und welche zusätzlichen Noxen sind wirksam? Für diese Frage sind Prävalenzstudien nicht ausreichend, man braucht Longitudinaluntersuchungen unter ausreichender Jodzufuhr.

7. Wenn eine ausreichend dosierte Jodprophylaxe die endemische Struma auch im höheren Lebensalter verhindert, gilt das auch für die "Folgekrankheiten «, also vor allem für die übermäßig häufigen autonomen Adenome bzw. multifokalen/disseminierten Autonomien der Schilddrüse?

Diese Fragen ließen sich ergänzen. Sie werden teilweise von manchen Arbeitsgruppen bearbeitet, wovon auch zwei Originalarbeiten in diesem Heft Zeugnis geben. Dennoch bleibt viel zu tun.

Prof. Dr. P. C. Scriba

Klinik für Innere Medizin

Medizinische Hochschule Lübeck

2400 Lübeck 1, Ratzeburger Allee 160 